\title{
PULSED POWER NETWORK BASED ON DECENTRALIZED INTELLIGENCE FOR RELIABLE AND LOW LOSS ELECTRICAL POWER DISTRIBUTION
}

\author{
Hisayoshi Sugiyama \\ Dept. of Physical Electronics and Informatics \\ Osaka City University, Osaka, Japan
}

\begin{abstract}
Pulsed power network is proposed for reliable and low loss electrical power distribution among various type of power sources and consumers. The proposed scheme is a derivative of power packet network so far investigated that has affinity with dispersion type power sources and has manageability of energy coloring in the process of power distribution. In addition to these advantages, the proposed scheme has system reliability and low loss property because of its intelligent operation performed by individual nodes and direct relaying by power routers. In the proposed scheme, power transmission is decomposed into a series of electrical pulses placed at specified power slots in continuous time frames that are synchronized over the network. The power slots are pre-reserved based on information exchanges among neighboring nodes following inherent algorithm of the proposed scheme. Because of this power slots reservation based on decentralized intelligence, power pulses are directly transmitted from various power sources to consumers with the least power dissipation even though a partial failure occurs in the network. The network operations with the proposed scheme is simulated to confirm the algorithms for the power slots reservation and to evaluate the power network capacity.
\end{abstract}

\section{Introduction}

Smart grid technologies have been investigated for the purpose to enhance efficiency and reliability of current power systems with smooth integration of renewable and alternative energy sources [1]. As one of the basics of this upcoming power grid, power packet transmission is proposed to distribute electric power over the consumers [2, 3, 4]. This scheme follows conventional data packet transmission where each fragment of data is transmitted individually with information including the addresses of its source and destination. Various data packets with different addresses share the same transmission path avoiding their mutual collisions.

Similar to the data packet networks, the power networking with power packets has advantages as follows. First, It has affinity with dispersion type power sources such like photovoltaic power generations and wind turbine generations. In current power systems, reverse power flow from dispersion type sources causes problems including voltage fluctuations that degrade power line quality to neighboring consumers [5]. In contrast, reverse power flow with power packets does not cause such problems because of collision avoidance executed among power fragments in power lines.

Second, It has manageability of energy coloring in the process of power distribution. Energy coloring means the attachment of associated information to each power fragment in addition to its source and destination $[6,7]$. The additional information includes power rate of the fragment, the type of its energy source, and the source location. This informa- 
tion assists consumers to select the electric power of the lowest charge, or to select the attribute of energy source concerning greenhouse gas emissions or such global scale problems.

In this paper, as a derivative of the power packet network, pulsed power network is proposed. In addition to the advantages inherent in the power packet networking above mentioned, the proposed scheme has inherent system reliability against a partial failure of the network, and has low loss property throughout the process of power distribution.

In the proposed scheme, power transmission is decomposed into a series of electric pulses placed at specified power slots in continuous time frames that are synchronized over the network. The power slots are pre-reserved throughout the transmission path from the power source to the consumer. This power slots reservation and preceding determination of the optimum transmission path and the power slot positions are performed by individual nodes of the source, destination, and intermediate routers. Their operations follow algorithms inherent in the proposed scheme with information exchanges among neighboring nodes.

These elemental operations of individual nodes with their localized collaborations configure decentralized intelligence over the network. Because no central supervisor stays in this intelligence, the operation of power distribution is reliable even when a partial failure occurs in the network. In addition, because of the foregoing power slot reservations performed by the network intelligence, power pulses are directly transmitted to consumers without any energy storage and forwarding at intermediate routers. This direct relaying at routers keeps power dissipation minimum throughout the power transmission path.

The fundamentals of the pulsed power network was already introduced in my previous work [8] as an approach to the power packet networking. However, the details were omitted of the decentralized intelligence of nodes in relation with the system operations. Moreover, based on the specified intelligence, the actual system performances were not evaluated by pertinent simulations. This paper focuses on these essentials of the proposed pulsed power network.
In section 2, the basics of the proposed pulsed power network is described with an example of the network configuration. In section 3, decentralized intelligence of the network is explained that is the basis of the reliability and low loss property of the proposed scheme. In section 4 , the performances and the reliability of the pulsed power network are evaluated by computer simulations. In the final section, summary of this paper and residual subjects for further study are described.

\section{Basics of pulsed power network}

The proposed scheme of pulsed power network is based on the synchronized frame structure consisting of power slots where electric pulses are located. These pulses are directly transmitted to consumers through power routers on the transmission path. In addition, power gateway connects this network with adjacent ones storing redundant power generated by inner and outer power sources. In this section, these basics of the pulsed power network are explained.

\subsection{Network configuration}

An example configuration of the proposed pulsed power network is shown in Fig. 1. This example consists of end users A to G, power routers $\mathrm{H}$ to $\mathrm{K}$, and a power gateway. Among end users, $\mathrm{A}$ and $\mathrm{F}$ with gray color are assumed to be power sources that reversely transmit power to the network through the router $\mathrm{H}$ and $\mathrm{K}$, respectively. Other end users consume electric power supplied from neighboring power routers $\mathrm{H}$, I, or $\mathrm{K}$. These energy flows are controlled by power routers $\mathrm{H}$ to $\mathrm{K}$ with their power relaying. The power gateway connects this pulsed power network with adjacent network converting the form of electric power flow if necessary. This gateway also stores redundant power supplied from both networks.

These nodes in the network are connected by power communication links. As shown in Fig. 1, each of the links consists of two transmission media: a power link and a communication link. The power link conducts the series of electric pulses, i.e., the power itself. On the other hand, the communication link conducts signals for the power transmission processing, i.e., the routing information 
and other control messages for the network operation. The communication links can be established by optical cables bundled with the power links as shown in the figure, or by other communication media so far investigated for the basis of smart grid operations[1]. In any case, signal transmission rate is assumed to be high enough that their transmission delay do not affect the operation of the power network itself.

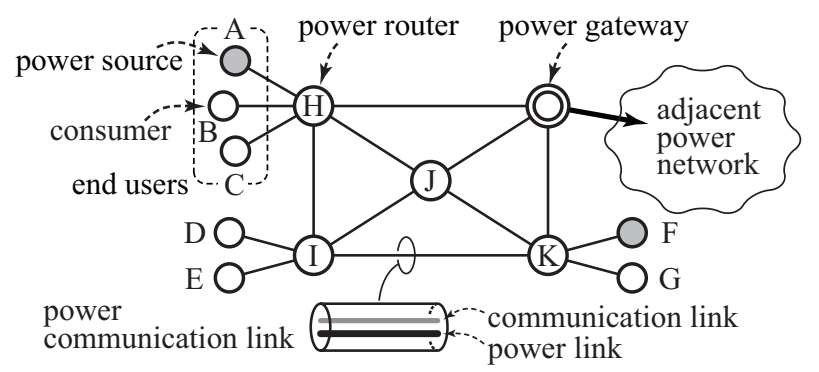

Figure 1. An example configuration of pulsed

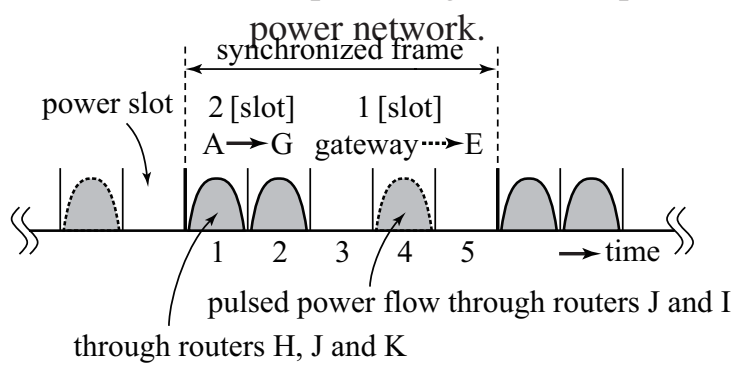

Figure 2. An example of synchronized frame structure and pulsed power flows.

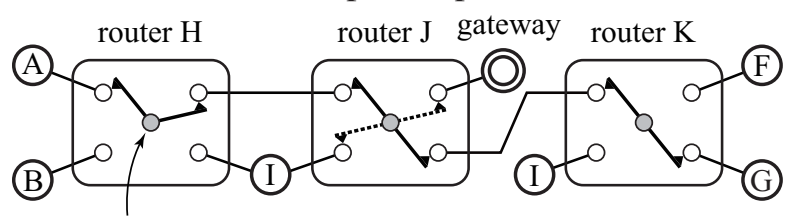

relay that connects two terminals

during specified power slots in successive frames

Figure 3. An example of direct relaying of power pulses in power routers.

\subsection{Synchronized frame structure}

In the proposed pulsed power network, time axis is divided into frames of equivalent length and each frame is equally subdivided into $N$ power slots.
Each frame is synchronized among all nodes in the network ${ }^{1}$. An example of this synchronized frame structure is shown in Fig. 2 where $N$ equals 5.

This figure also indicates pulsed power flows on the synchronized frames. From now on, a power flow means a mass of energy transmission (measured in joule) from a power source to a consumer that continues for a predetermined time with a constant intensity (in watt). Whereas, the pulsed power flow means the decomposed power flow by electric pulses transmitted at the same positions of power slot in successive frames until the power flow itself is completed.

The energy conveyed by each electric pulse is assumed to be constant. Therefore, the number of power slots in one frame used for the pulsed power flow specifies the intensity of the power transmission. Because of this reason, from now on, the intensity of power transmission is denoted by the number of power slots in one frame. For example, Fig. 2 shows two power flows from node A to node $\mathrm{G}$ (solid contours), and from the power gateway to node $\mathrm{E}$ (dashed contour) ${ }^{2}$. Their intensities are two slots and one slot, respectively.

\subsection{Power router}

In the proposed pulsed power network, each transmission path for the pulsed power flow is established through direct relaying by cascading routers. Figure 3 shows an example of this operation by power routers $\mathrm{H}, \mathrm{J}$, and $\mathrm{K}$, selected from Fig. $1^{3}$. They configure a power transmission path from the power source $A$ to the consumer $\mathrm{G}$ by relay switches that are schematic representations of relay circuits constructed with power semiconductors.

Within the first and second slots in each synchronized frame, as indicated in Fig. 2, each relay switch connects appointed terminals. Therefore, within these time slots, the power pulse flows from the source node A to the consumer G. The intensity of transmitted power through this path is two slots a frame. Whereas, the dashed relay conductors in the router $\mathbf{J}$ indicates the simultaneous pulse flow from the power gateway to consumer $\mathrm{E}$ beyond the

\footnotetext{
${ }^{1}$ This network synchronization can be established somehow. For example, assuming that the length of each power slot is in millisecond order, adequate network synchronization is available by using GPS time signals[9].

${ }^{2}$ The location of these nodes are indicated in Fig. 1, and this frame structure is observed at power router J.

${ }^{3}$ According to Fig. 1, router $\mathrm{H}$ and $\mathrm{K}$ actually have six and five relay terminals, respectively. However, some of them are omitted for the simplicity.
} 
router I. As Fig. 2 indicates, this pulse flows within the fourth slot. The intensity of this pulsed power flow is one slot a frame.

\subsection{Power gateway}

As mentioned before, the role of the power gateway includes electric power form conversion and storage of redundant power supplied from inner and outer networks. With these operations, the power gateway provides the pulsed power network with following three aspects.

First, the whole network can be constructed as an integration of subnetworks connected by power gateways. All of the network operations are restricted within each subnetwork. This isolated networking saves calculation cost of each node that must count in current status of every other nodes in the network. Whereas, essential information of outer networks are merged in the power gateway that includes the volume of surplus power of centralized generation plants with each of their attributes. According to this information, each consumer is able to require power supply from particular power plant in outer areas.

Second, hierarchical network configuration becomes possible consisting of network layers of various aspects with different power line voltages. For example, each end user indicated in Fig. 1 is actually a power gateway to HAN (home area network)[10] that is the lowest layer power network. On the other hand, the adjacent power network possibly be a higher layer network that conveys high voltage electric power from distant power plants. Because of the power form converting the gateway operates, the form of outer area network possibly be the conventional one or that of pulsed power network itself.

Third, concentrated electric power storage can be provided for end users that have dispersion type power sources. In conventional power systems, these users usually prepare their own rechargeable batteries for stabilized power consumption and for unexpected power outages. On the other hand, in the proposed scheme, power gateway of each subnetwork has bulk power storage for redundant power of inner and outer networks. Because this bulk storage stores electric power generated by end users with their individual identities carried by energy coloring, the storage can replace the rechargeable batteries of end users. This concentrated electric power storage in power gateways possibly save the installation cost of dispersion type power sources.

\section{Decentralized system intelligence}

In the operation of pulsed power network, power loss at power routers is kept low enough because of foregoing reservation of power slots throughout each transmission path, and of direct relaying of the routers without any store and forward process of power pulses. In addition, the network operation continues even though some partial failures occur at the routers and power communication links.

These advantages of the pulsed power network are based on decentralized intelligence as an integration of elemental behaviors of power sources, consumers, power routers, and a power gateway. Their behaviors follow two kinds of algorithm. One for the update process of power transmission capacity table (or PTC table), and the other for the reservation process of power slots throughout the power transmission path. In this section, algorithms for these processes are described ${ }^{4}$.

\subsection{Power transmission capacity table}

The PTC table is the essential component for the operation of pulsed power network. Every node in the network constructs its own PTC table individually and updates frequently in a decentralized scheme.

When a power source intends to transmit power pulses to some consumer, the PTC table of the source node shows which slots are currently available for the power transmission to the destination and which adjacent node is the next hop on the most preferable path to the destination. According to this information, the power source begins to reserve the power slots throughout the transmission path to the destination. PTC tables of intermediate power routers are also necessary for this reserva-

\footnotetext{
${ }^{4}$ These algorithms are based on DSDV[11, 12] and Synchronized QoS routing[13, 14] for wireless ad hoc networking. Their aspects are modified for especial requirements of wired pulsed power networking.
} 
tion process and for table update of every node in the network. An example of the PTC table is shown in Fig. 4.

This example shows the PTC table owned by node $\mathrm{H}$ that is a power router indicated in Fig. 1. When a PTC table construction is completed, the table consists of entries that indicate current state of transmission paths to every destination node in the network. In Fig. 4, the entries are indicated by four lines.

\begin{tabular}{|c|c|c|c|c|c|c|c|c|c|c|c|c|}
\hline \multirow{2}{*}{ dst } & \multirow{2}{*}{ nxt } & \multirow{2}{*}{ hop } & \multirow{2}{*}{ slt } & \multirow[b]{2}{*}{ seq } & \multicolumn{8}{|c|}{ available slots } \\
\hline & & & & & 1 & 2 & 3 & 4 & 5 & 6 & 7 & 8 \\
\hline $\mathrm{A}$ & $\mathrm{A}$ & 1 & 6 & 12 & $x$ & 0 & 0 & $\bigcirc$ & 0 & $x$ & 0 & 0 \\
\hline $\mathrm{G}$ & I & 2 & 4 & 24 & 0 & 0 & $x$ & 0 & $x$ & 0 & $x$ & $x$ \\
\hline $\mathrm{H}$ & $\mathrm{H}$ & 0 & 8 & 10 & 0 & 0 & 0 & 0 & 0 & 0 & 0 & 0 \\
\hline $\mathrm{K}$ & $\mathrm{J}$ & 2 & 5 & 20 & $x$ & 0 & $x$ & 0 & 0 & 0 & 0 & $x$ \\
\hline
\end{tabular}

Figure 4. An example of PTC table owned by node $\mathrm{H}$.

In each entry, the destination is denoted by 'dst'. 'nxt' and 'hop' means the next hop node and the hop count to the destination, respectively. (From now on, "the entry points A over B" means "the entry has node A as its destination and B as the next hop".) 'slt' indicates the channel capacity by number of available slots for the power transmission. 'seq' means the sequence number that indicates freshness of the entry. 'available slots' specify which slots are currently available (denoted by ' $\bigcirc$ ') to transmit power pulses to the destination. In this example, the number $N$ of slots equals 8 in one frame.

At the beginning of a PTC table construction, the table consists of only one entry: the primary entry. This primary entry points the owner node itself. The initial value of 'seq' is set to zero and is increased periodically. An example is shown in Fig. 4 where the primary entry with gray color points the owner node $\mathrm{H}$ itself. When adjacent nodes mutually refers the contents of their PTC tables, the primary entry of the owner is added to PTC tables of others as regular entries. These regular entries are spread more over the network. At this spreading, every node examines the freshness of the referred entry by its value of 'seq'.

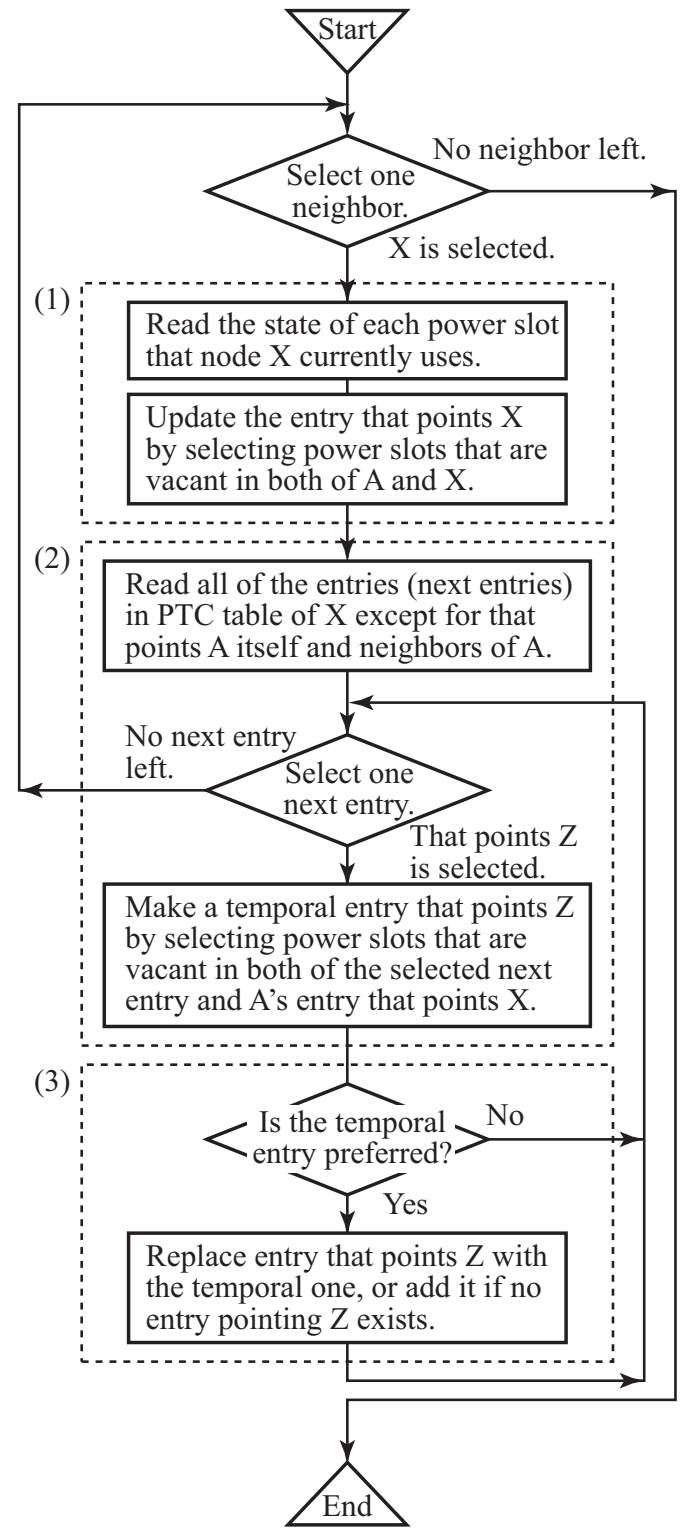

Figure 5. Algorithm for PTC table update. The owner node of this table is denoted by 'A'.

The update algorithm of the PTC table for each node is indicated by Fig. 5 as a flowchart. In this chart, the owner node of the table is denoted by 'A' that executes this algorithm. The process begins with the selection of one neighbor node around A. The selected node is denoted by ' $\mathrm{X}$ '. Then, successive processes run as follows (each number corresponds with the tag attached to a dashed frame in the figure). 


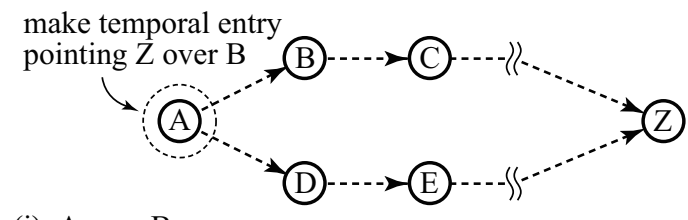

(i) $\mathrm{A} \longrightarrow \mathrm{B}$

\begin{tabular}{|c|c|c|c|c|c|c|c|c|c|c|c|c|}
\hline dst & nxt & hop & slt & seq & 1 & 2 & 3 & 4 & 5 & 6 & 7 & 8 \\
\hline B & B & 1 & 5 & 10 & $\times$ & $\bigcirc$ & $\times$ & $\bigcirc$ & $\bigcirc$ & $\bigcirc$ & $\times$ & $\bigcirc$ \\
\hline
\end{tabular}

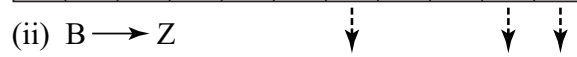

\begin{tabular}{|c|c|c|c|c|}
\hline \multicolumn{2}{|l|}{\begin{tabular}{|l|l|l|l|l|} 
dst & nxt & hop & slt & seq \\
\end{tabular}} & 2 & 3 & \\
\hline
\end{tabular}

\begin{tabular}{|l|l|l|l|l|l|l|l|l|l|l|l|l|}
\hline $\mathrm{Z}$ & $\mathrm{C}$ & 4 & 4 & 18 & $\bigcirc$ & $\bigcirc$ & $\mathrm{X}$ & $\times$ & $\bigcirc$ & $\bigcirc$ & $X$ & $\times$ \\
\hline
\end{tabular}

(iii) $\mathrm{A} \longrightarrow \mathrm{Z}$ ì

\begin{tabular}{|c|c|c|c|c|c|c|c|c|c|c|c|c|}
\hline dst & nxt & hop & slt & seq & 1 & 2 & 3 & 4 & 5 & 6 & 7 & 8 \\
\hline $\mathrm{Z}$ & $\mathrm{B}$ & 5 & 3 & 20 & $\mathrm{X}$ & $\bigcirc$ & $\mathrm{X}$ & $\times$ & $\bigcirc$ & $\bigcirc$ & $\mathrm{X}$ & $\mathrm{X}$ \\
\hline
\end{tabular}

Figure 6. Example of temporal entry construction that points $\mathrm{Z}$ over $\mathrm{B}$ from node $\mathrm{A}$.

(1) In this stage, node A update the entry that points the selected neighbor X. First, node A refers the state of each power slot that X currently uses. Then, A updates its own entry that points $X$ by selecting power slots that are vacant in both of $\mathrm{A}$ and $\mathrm{X}$.

(2) In this stage, node A makes a temporal entry that points nonadjacent nodes over X. First, A reads all of the entries in PTC table of X except for that points $\mathrm{A}$ itself and neighbors of $\mathrm{A}$. These entries are called next entries. Among them, one next entry that points ' $Z$ ' is selected (or, if no next entry left, A returns to the start point). Then, A makes a temporal entry that points $\mathrm{Z}$ by selecting power slots that are vacant in both of the selected next entry and A's entry that points $\mathrm{X}$.

(3) In this final stage, node A updates entry that points Z. First, A decides whether the temporal entry pointing $\mathrm{Z}$ is preferable to the existing entry pointing the same node ${ }^{5}$. If preferable, A replaces the existing entry with the temporal one. Or, if no entry pointing $\mathrm{Z}$ exists, $\mathrm{A}$ adds the temporal entry to its PTC table. Then, A returns to the middle point of the previous stage as the figure indicates.

According to this flowchart, an example process of PTC table update is illustrated by Fig. 6. In this figure, node A is assumed to update its PTC table and be located with two neighbors: nodes $\mathrm{B}$ and $\mathrm{D}$. Beyond these neighbors, other nodes exist including $\mathrm{C}, \mathrm{E}$, and $\mathrm{Z}$ connected with each other as the figure shows.

In this example process, the entry is selected to be updated that points node $\mathrm{Z}$ in the PTC table of node $\mathrm{A}$. In addition, among neighbors of $\mathrm{A}$, node $\mathrm{B}$ is assumed to be selected at the beginning of the update process. Then, the first and second stages above mentioned are executed as follows.

(1) Node A updates the entry that points B. First, Node A reads the state of each power slot that node B currently uses. Then, A updates the entry pointing $\mathrm{B}$ by selecting power slots that are vacant in both of node $A$ and $B$. The example of this result is indicated in Fig. 6 as "(i) $A \rightarrow B$ "6 .

(2) Node A makes the temporal entry that points nonadjacent nodes $\mathrm{Z}$ over B. For this purpose, $A$ reads the next entry that points $Z$ in PTC table of $B$. The example of this result is indicated in Fig. 6 as "(ii) B $\rightarrow Z$ ". Then, A makes the temporal entry that points $Z$ by selecting power slots that are available in both of the entries (i) and (ii) above mentioned. This selection is expressed by dashed arrows in the figure. The result of this temporal entry is indicated in Fig. 6 as "(iii) $\mathrm{A} \rightarrow \mathrm{Z}$ '.

In the final stage (3), node A decides whether the temporal entry "(iii) $\mathrm{A} \rightarrow \mathrm{Z}$ " is preferable to the existing entry pointing the same node $Z$. The primary standard for this decision is the freshness of the entries indicated by the number of 'seq'. If this number of the temporal entry is smaller than that of existing one, the former is decided to be preferable. Or, if these numbers are equivalent to each other, succeeding decision standards for the preferable entry are as follows.

1. Which entry has a smaller 'hop'? This standard means that the entry indicating a shorter path to the destination is preferable to the other.

2. Which entry has a larger number 'slt' of available slots? This standard means that the entry

\footnotetext{
${ }^{5}$ Conditions for this decision are described later.

${ }^{6}$ In figures (i), (ii), and (iii), $N$ is set to 8 and only the entry concerned is extracted from PTC table of node A or B. In addition, values of 'nxt', 'hop', and 'seq' are set appropriately according to the protocol of DSDV[11, 12].
} 
indicating the more capacity of power transmission to the destination is preferable to the other.

The first standard appreciates the power saving against Joule loss during the power transmission. On the other hand, the second one appreciates the availability of power slots to the destination. Concerning the average network performance within a long time, the hop count and the number of available slots to the same destination may have a noticeable correlation. However, frequent update of the PTC tables according to one of the standards possibly causes different network performance. This difference is investigated by simulations in the next section.

As described above, the update of each PTC table is executed in pure decentralized scheme. Because of this decentralized aspect of the essential operation, the pulsed power network has the inherent system reliability of the electric power distribution that is especially desirable from the view point of disaster prevention in urban areas.

\subsection{Power slots reservation process}

When a power source in the pulsed power network intends to transmit a power flow to a consumer, the source node pre-reserves the power slots throughout the transmission path to the destination. The number of the reserved slots in one frame corresponds with the intended intensity of the pulsed power flow.

For this power slots reservation process, and for the succeeding pulsed power flow to the destination, control messages are exchanged among source node, destination, or intermediate power routers. The transmission path for the control message is established by cascading next hop nodes that begins at the sender node. The next hop node is indicated as 'nxt' on the entry pointing the receiver node in the PTC table of each node. The control messages are as follows.

RESERVE This message is sent from the power source to the destination through intermediate routers directing these nodes to reserve specified power slots with appropriate schedule for the power pulse relaying.

REPLY This message is inversely sent from the destination to the power source indicating that the power slots reservation is accomplished throughout the power transmission path. If a source node receives this message, it is assured of the reservation and begins the transmission of power pulses at the reserved positions of power slots.

ERROR If the power slots reservation process fails at some node within the power transmission path, the node send this message ERROR to the source node. The arrival of this message informs the source node that the intended power slots reservation finishes unsuccessfully. The reason of this trouble occurring possibly be conflict with the same reservation message from another power source, or temporal mismatch between the PTC tables of neighboring nodes on the transmission path.

\begin{abstract}
ADEQUATE At the end of power flow transmission, this message is inversely sent from the destination to the power source indicating that the expected power flow reception by the destination is successfully finished.
\end{abstract}

RELEASE When the power source is assured of the accomplishment of the intended power flow, it sends this message to the destination through intermediate routers directing these nodes to release power slots that are occupied for the specified pulsed power flow.

With these control messages exchanged among other nodes, the power source, destination, and intermediate power routers perform their particular behaviors for the power slots reservations. Among these behaviors, that of the destination and power routers are rather simple. For example, a power router reserves its power slots or releases them according to RESERVE or RELEASE, respectively. In case of some trouble occurring, it sends ERROR to the source node. Because of this reason, only the algorithm that the power source follows is explained. 


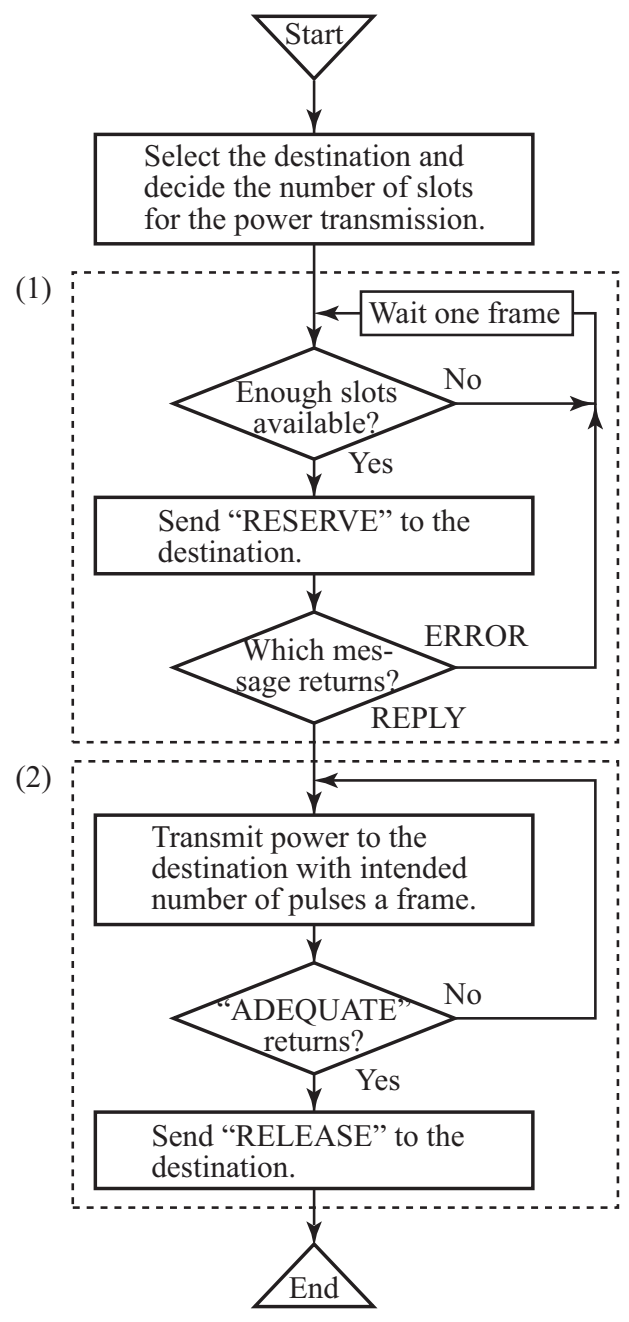

Figure 7. Algorithm for power source to reserve power slots and transmit power pulses to the destination.

Figure 7 shows the algorithm for each power source that intends to reserve power slots to a destination. This algorithm also includes a part for the succeeding process of the pulsed power transmission through the reserved power slots. First, the source node selects the destination and decides the number of power slots for the pulsed power flow $^{7}$. Then, as the figure shows, the process runs as follows (each number corresponds with the tag attached to a dashed frame in the figure).

(1) This first part of the algorithm indicates that for the power slots reservation itself. According to the selection of the destination and decision of the number of slots for the power transmission, the source node refers its own PTC table and confirms if enough slots are available to the destination.

If the result is "No", the source node waits a frame passes and refers again its PTC table. This is because the interval of the table updates must be longer than the frame duration time.

In contrast, if the result is "Yes", the source node selects power slots among available ones and send the message "RESERVE" to the destination. This signal reserves selected slots throughout the power transmission path to the destination.

If some trouble occurs during this power slots reservation process, the source node receives "ERROR". In this case, the source node returns to the beginning of this stage as the figure shows. Then, after a frame passes, the process restarts from the PTC table referring.

Or, if the power slot reservation is accomplished and the message "REPLY" returns from the destination, the source node proceeds to the next stage.

(2) This second part of the algorithm indicates that for the pulsed power transmission through the reserved power slots on the transmission path to the destination.

This pulsed power transmission is triggered by "REPLY" and continues until the message "ADEQUATE" returns from the destination. The source node observes repeatedly if this message returns.

If the source node receives "ADEQUATE", this means the expected power flow reception by the destination is successfully finished. In this case, the source node transmits "RELEASE" to the destination. This message releases the reserved power slots throughout the transmission path.

\section{Network performance simulation}

System performance of the power network with the proposed scheme is estimated by computer simulations on an example model of pulsed power network. The estimated points are as follows:

\footnotetext{
${ }^{7}$ This process of the source node depends on the preceding information of power demand from consumers. Therefore, there must be some previous process that is triggered by the consumers. The details of this subject is discussed in my recent work[15].
} 
1. The simulation of the PTC table construction and update process at every node in the adopted network model, and simulation of the integrated network operation based on the PTC tables.

2. The evaluatoin of the power network capacity that is defined as the maximum possible volume of simultaneous power transmissions among power sources and consumers over the network.

3. The confirmation of the network reliability against a partial failure of the network. The reliability is based on the pure decentralized aspect of the pulsed power network operation.

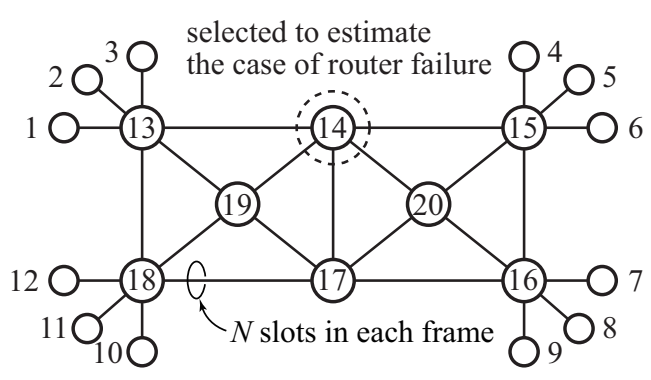

Figure 8. Simulation system model.

\subsection{Simulation system model}

Figure 8 shows the simulation system model with twelve end users (node $1 \sim 12$ ) and eight power routers (node $13 \sim 20$ ). End users are classified randomly into power sources and consumers half and half as described later. Among power routers, node 14 , as indicated by a dashed circle, is selected to estimate the case of router failure.

The intensity of each pulsed power flow from a source node to a consumer is assumed to be one slot each frame. This is because one slot reservation has more possibility than simultaneous multiple slot reservations. Therefore, as the second estimate point above mentioned, the evaluation of the power network capacity approaches the proper value with the one slot reservation for each pulsed power flow. During the simulation, multiple slot reservation may occur between the same pair of power source and consumer by accumulating the one slot reservations. In this case, power transmission paths for the reservations are not necessarily the same with each other.

The simulation procedure is as follows:

1. PTC tables of all nodes are initialized. This means that each node constructs its own PTC table consisting of only the primary entry that points the owner node.

2. According to the algorithm indicated in Fig. 5, each node updates its own PTC table referring other tables of neighboring nodes. This process repeats until every node completes its PTC table with entries that point all nodes in the network.

3. A half of the end users are selected randomly to be the transmitters of pulsed power flow and the residual ones are assumed to be the receivers.

4. Among these end users, a pair of transmitter and receiver is selected randomly and its power transmission path is determined according to the PTC tables of the transmitter and successive power routers.

5. Throughout the power transmission path, number $K$ of power slots are reserved ${ }^{8} . K$ is the intensity of the pulsed power flow and is set to one as mentioned above. Succeedingly, PTC tables of all nodes are updated counting in just occupied power slots by this reservation.

6. The above stages 4 and 5 are repeated until the selected transmitter cannot find the transmission path to the reciever any more in its PTC table ${ }^{9}$

7. Finally, the power network capacity is determined as the number of the transmission paths established multiplied by the intensity $K$ (equals one) of each pulsed power flow.

This result of the power network capacity varies among many of the simulation runs. Therefore, by

\footnotetext{
${ }^{8}$ The algorithm for the reservation indicated in Fig. 7 is not traced in the simulations. This requires the assumption that PTC table updates and control message transmissions are so fast that troubles do not occur in the power slots reservations.

${ }^{9}$ Because the intensity $K$ of the pulsed power flow is set to one, this means that the entry that points the receiver has no available slots at the time.

${ }^{10}$ Because this total power transmission is associated with the sccess rate that 100 percent is not certified, the term "power network capacity" is not used in further descriptions.
} 
averaging these results, success rate of the power slots reservation is derived at each volume of the total power transmission ${ }^{10}$. This averaged simulation result is shown in the next subsection.

\subsection{Simulation results}

Figure 9 shows the success rate of the power slots reservation versus the total power transmission. The horizontal axis represents the total power transmission estimated by number of power slots. Whereas, the vertical axis represents the success rate of power slots reservation. Number $N$ of power slots is set to 20. There are two groups of lines that indicate this success rate: the group where no failure occurs among routers, and the group where the router 14 fails and does not work throughout the network operation.

Each group consists of three types of lines: solid line, chain line, and dashed line. The solid line and chain line represents the results of the simulation where power transmission path reservation is based on the PTC tables of nodes. The former result regards the number of available slots first when PTC table is updated, and the hop count to the destination is secondary regarded. Whereas, the latter result only regards the hop count at the table update. On the other hand, the dashed line represents the simulation results where each transmission path is selected from many times of random path search over the network. Though this method cannot be adopted in actual network operations, nearly optimum path is found at each power transmission. The latter result is referred from my previous work[8].

As mentioned above, these lines indicate the total power transmission with allowable success rate of power slots reservation. For example, if 80 percent of success rate is allowable, the total power transmission is roughly 30 slots provided no failure occurs among routers. This means that 30 slots of power transmission between pairs of randomly selected power source and consumer, or 30 pulsed power flows between them with one slot intensity in this case, will be established with 80 percent of probability ${ }^{11}$.

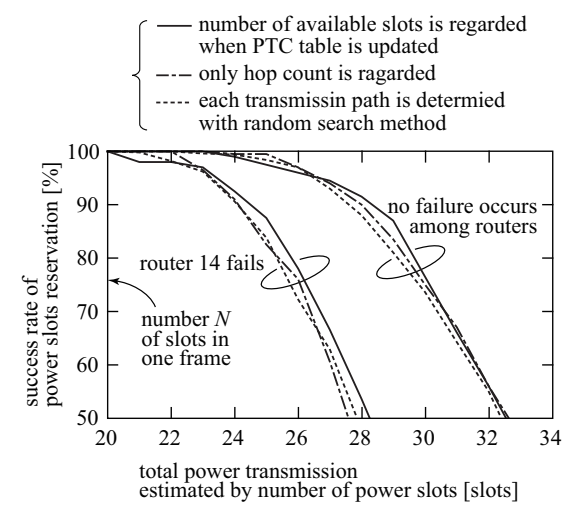

Figure 9. Success rate of power slots reservation versus total power transmission when number $N$ of slots is set to 20 in each frame.

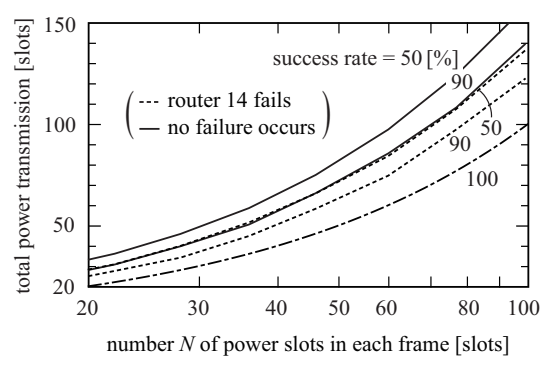

Figure 10. Power network capacity at specified success rate versus number $N$ of power slots.

Figure 9 shows three points as follows:

1. All of the lines reach 100 percent of success rate at the left end of the horizontal axis. This left end is set to 20 and is equal to the number of power slots $N$ in one frame. This confirms that 100 percent of success rate is always obtained provided the total power transmission does not exceed the number of slots $N$. This is easily understandable because every power transmission can be allocated to individual power slots under this condition.

2. Over this piont of total power transmission, the success rate of power transmission degrades and the gradient depends on whether the failure of router 14 occurs or not. If the failure occurs, the gradient becomes sharp and the success rate falls to 50 percent fast. On the other hand, if no router failure occurs, the gradient is modest and the success rate remains relatively high to some range of the total power transmission. This

\footnotetext{
${ }^{11} 100$ percent of success rate may not indispensable in actual operations of power networks. Because each consumer prepares a power storage in his power gateway, as described before in subsection 2.4, power supply fluctuations are smoothed to some degree by this storage.
} 
shows that the simulated network operation is not so differ from that expected, and that the network well operate even though a router failure occurs at some cost of the success rate degradation.

3. The difference is recognized between the simulation results that depend on the two kinds of standard. The standards are the number of available slots and the hop count to the destination when PTC table is updated. Though the difference is recognized as small, the advantage of the former standard: the number of available slot is confirmed. In addition, because the difference is recognized small between these simulation results depending on PTC tables and that depending on random search method for the power transmission path determination, the proposed scheme based on PTC tables is confirmed as almost optimal method to perform most effective networking of power distribution.

As shown in Fig. 9, when the success rate is specified, improvement of the total power transmission is possible by reconstruction of network configuration or by increase of number $N$ of power slots in each frame. Among these factors, the more feasible one is to increase the number $N$ of power slots. Because the total power transmission equals $N$ at 100 percent of the success rate, the total power proportionally improves as $N$ increases. At other success rates, this relationship between the total power transmission and the number $N$ of power slots is extracted from the simulations where the number of available slots is regarded at PTC table updates. The results are shown in Fig. 10.

The horizontal axis represents the number $N$ of power slots in each frame, and the vertical axis represents the total power transmission. Success rates of 100,90 , and 50 percent are indicated as the parameters. Dashed lines indicate the case of failure of power router 14 . Whereas, solid lines indicate the case with no failure occurring. Chain line incidates the case when success rate equals 100 persent. In this case, network configuration does not affect the result.

As this figure shows, the total power transmissions increase almost proportionally to $N$ at indicated values of success rate. However, concerning this approach of $N$ increase, there must be limita- tions as follows.

1. The increase of $N$ causes the decrease of each power slot duration. Therefore, assuming that the intensity of the pulsed power flow is specified, the peak voltage of each power pulse must be expanded. From the view point of electrical signal processing and conducting throughout the power transmission path, some limitations must exist on this peak voltage.

2. The decrease of each power slot duration requires more accurate synchronization among frames and power slots over the network. Or, more length of guard intervals must be located between adjacent power pulses. Even though GPS time signals are available for the synchronization, limitation exist on the time precision.

\section{Conclusion}

Pulsed power network is proposed for reliable and low loss electrical power distribution among various type of power sources and consumers. In addition to the advantages of power packet networks that are affinity with dispersion type power sources and manageability of energy coloring in the process of power distribution, the proposed scheme has system reliability and low loss property because of its intelligent operation performed by individual nodes and direct relaying by power routers. The details of these aspects of the proposed scheme are explained and the network performances are confirmed by simulations.

In further studies, following subjects must be investigated.

1. Mutual cancellation of power pulses must be investigated that alleviates the restriction of the power network capacity of the proposed scheme. This alleviation is evaluated in my previous work[8] and noticeable improvement is confirmed. In further studies, an algorithm that deals with the mutual cancellation must be designed in relation with the PTC table constructions.

2. Dynamical aspects of the actual power network operations and other parameters must be considered in further simulations such as delay estima- 
tions of control signals. At the simulations, reasonable designs of synchronized frame length, number of power slots $N$, network topology, and system dimensions must be counted in.

3. Along with these sophisticated simulations, characteristics of the proposed power network must be quantified that includes the efficiency of network operations, the evaluation of power losses, and the estimation of reliability against system troubles. In addition, to realize this proposed network, some practical appliactions must be investigated based on their cost estimation of construction and preservation in comparison with conventional power systems.

\section{References}

[1] V. C. Gungor, et al., "Smart Grid Technologies: Communication Technologies and Standards", IEEE Trans. Industrical Informatics, Vol.7, No.4, pp. 529-539, 2011.

[2] J. Toyoda, H. Saitoh, "Proposal of an OpenElectric-Energy-Network (OEEN) to Realize Cooperative Operations of IOU and IPP,' EMPD'98, Mar. 1998.

[3] T. Takuno, M. Koyama, and T. Hikihara, "In-Home Power Distribution Systems by Circuit Switching and Power Packet Dispatching," SmartGridComm, pp. 427-430, Oct. 2010.

[4] V. Krylov, D. Ponomarev, and A. Loskutov, "Toward the Power InterGrid," IEEE Energy Conference, pp. 351-356, Dec. 2010.

[5] H. Hatta, M. Asari, and H. Kobayashi, "Study of Energy Management for Decreasing Reverse Power Flow from Photovoltaic Power Systems," SAE2009, pp. 1-5, Sept. 2009.
[6] T. Kato, et al., "Appliance Recognition from Electric Current Signals for Information-Energy Integrated Network in Home Environmens," Int. J. Assistive Robotics and Systems, Vol.10, No.4, pp. 5160, 2009.

[7] K. Saito and Y. Okabe, "Quality-Aware Energy Routing Toward On-Demand Home Energy Networking," CCNC2011, Jan. 2011.

[8] H. Sugiyama, "Direct Relayed Power Packet Network with Decentralized Control for Reliable and Low Loss Electrical Power Distribution," GCCE2013, pp. 32-36, Oct. 2013.

[9] R.F.M. Brandao, J.A.B. Carvalho, and F.M. Barbosa, "GPS Synchronized Measurements in Power Systems State Estimation: An Overview," UPEC '06, Sept. 2006.

[10] V. Namboodiri, et al., "Toward a Secure WirelessBased Home Area Network for Metering in Smart Grids," IEEE Systems Journal, Vol.8, No.2, pp. 509-520, 2014.

[11] C. E. Perkins and P. Bhagwat, "Highly Dynamic Destination-Sequenced Distance-Vector Routing (DSDV) for Mobile Computers," ACM SIGCOMM'94, pp.234-244, 1994.

[12] H. Sugiyama, T. Tsujioka, and M. Murata, "Ad Hoc Network Simulator Based on DSDV Routing Method," Memoirs of the Faculty of Engineering, Osaka City Univ., pp.43-58, 2002.

[13] C. R. Lin and J. S. Liu, "QoS Routing in Ad Hoc Wireless Network", IEEE J. Select. Areas Commun., Vol.17, No.8, pp. 1426-1438, 1999.

[14] H. Sugiyama, "Bandwidth Table Construction Regarding CDMA Interferences in Synchronized QoS Ad Hoc Networking," ISABEL2011, Oct. 2011.

[15] H. Sugiyama, "Power Distribution Protocol Among Power Plants and Consumers in Power Packet Networks," GCCE2014, Oct. 2014.

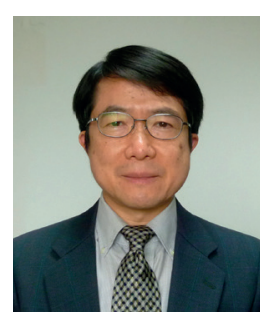

Hisayoshi Sugiyama graduated from the Nagoya University, Japan, in 1978. He received the Ph.D. degree from the same University in 1983. He joined the NTT Laboratories in 1983. Since 1994, he has been an Associate Professor at the Department of Physical Electronics and Informatics of the Osaka City University, Osaka, Japan. His interests include wireless ad hoc networking, multi-robot rescue systems, wireless power transmission, and smart grid power systems. 